\title{
Tormenta eléctrica: una manifestación inusual de ectasia coronaria
}

\author{
Electrical storm: an unusual manifestation of coronary ectasia \\ Luis M. González-Galván ${ }^{1 *}$, Diego Araiza-Garaygordobil', Jorge L. Vargas-Estrada ${ }^{1}$ y \\ Edgar Illescas-González \\ ${ }^{1}$ Unidad Coronaria; ${ }^{2}$ Departamento de hemodinámica. Instituto Nacional de Cardiología Ignacio Chávez, Ciudad de México, México
}

\section{Introducción}

La ectasia coronaria (EC) constituye una enfermedad difusa del vaso que causa una dilatación mayor a 1.5 veces en comparación a un segmento adyacente de arteria sana. La incidencia de la EC es del 0.3 al $4.9 \%$ en la población general' ${ }^{1}$. A pesar de su relativa frecuencia, la presentación clínica, el origen, el manejo y la prevención de la EC siguen siendo tema de debate e investigación médica continua ${ }^{2}$. Reportamos el caso de una presentación infrecuente de la EC: la tormenta eléctrica.

\section{Caso clínico}

Hombre de 42 años con antecedentes de diabetes mellitus tipo 2 de larga evolución y consumo intenso de tabaco durante 20 años. Acudió al Servicio de Urgencias de nuestro hospital por una historia de 24 horas de dolor precordial opresivo irradiado al hombro izquierdo y asociado a disnea. En su examen físico destacaban signos vitales: FC 84 lpm, TA 106/70 mmHg, FR $22 \mathrm{rpm}$ y $\mathrm{SpO}_{2} 86 \%$, así como la presencia de estertores subcrepitantes en ambas bases pulmonares. El precordio con ruidos cardiacos rítmicos, sin soplos u otras anomalías. El electrocardiograma de ingreso en ritmo sinusal, con una zona inactivable inferior (QS). Durante su revisión en urgencias presentó súbitamente diaforesis profusa y se documentó taquicardia ventricular (TV) (Figura 1), hemodinámicamente estable. Se inició tratamiento con amiodarona sin resolver el cuadro; por la persistencia de síntomas se llevó a cabo cardioversión eléctrica en tres ocasiones, siendo fallida. Se administró lidocaína intravenosa (i.v.), ante lo cual se obtuvo ritmo sinusal durante un lapso de 30 segundos, para nuevamente presentar TV. Se decidió realizar intubación orotraqueal y llevar al paciente a coronariografía diagnóstica, la cual reportó EC en las tres arterias principales, con importante carga trombótica (trombo TIMI i.v. en arteria coronaria derecha y trombo TIMI i.v. en circunfleja), así como flujo lento TIMI II (Figura 2). Se administró dosis intracoronaria de tirofibán y trombólisis in situ con tenecteplasa intracoronaria en la coronaria derecha (responsable del infarto). Se colocó balón intraaórtico de contrapulsación por la persistencia de TV y, encontrándose en sala, se realizó cardioversión eléctrica por cuarta ocasión, obteniéndose ritmo sinusal y mejoría en estado hemodinámico. El paciente fue trasladado a la Unidad coronaria, donde presentó mejoría durante las siguientes horas.
Correspondencia:

*Luis M. González Galván

E-mail: dr.galvan1206@gmail.com BY-NC-ND (http://creativecommons.org/licenses/by-nc-nd/4.0/).

Disponible en internet: 00-00-2019 Arch Cardiol Mex. 2020;90(2):224-226 www.archivoscardiologia.com 


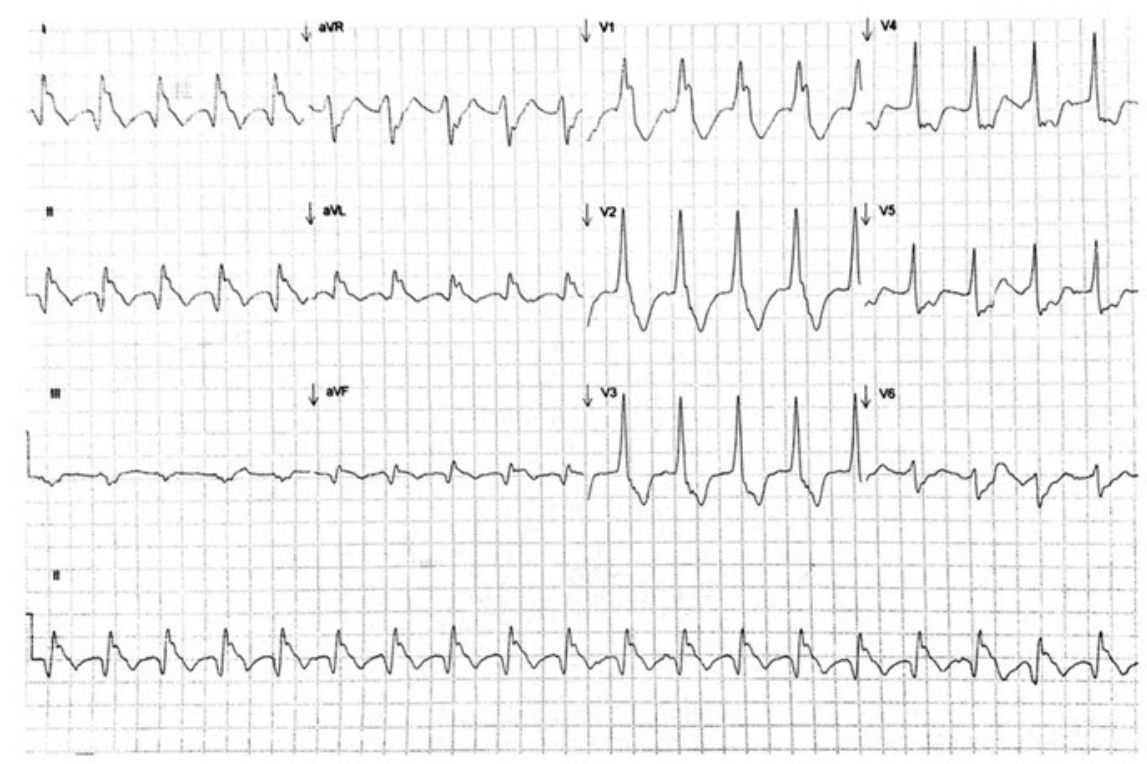

Figura 1. El ECG muestra una taquicardia de complejo ancho, monomórfica, con eje horizontal izquierdo y morfología de BRD, lo que sugiere que el sitio de origen de la taquicardia ventricular puede estar en los segmentos basales del septum interventricular del lado izquierdo.

\section{Discusión}

En 1761 Morgagni $^{3}$ describió por primera vez las características anatómicas de la EC en un paciente con sífilis, pero el término ectasia (del latín ectasis, dilatación) fue acuñado por Bjork en 1966. En más de la mitad de los casos, la EC se encuentra asociada a enfermedad coronaria aterosclerótica, pero en ocasiones menos frecuentes se ha relacionado a otras condiciones patológicas como exposición a herbicidas, enfermedad de Takayasu, poliarteritis nodosa, traumatismo o lesión directa vascular, etc ${ }^{5}$. Las tres arterias coronarias pueden ser afectadas por EC pero en el $75 \%$ de los pacientes es una arteria aislada la que se encuentra ectásica ${ }^{5}$. Markis, et al. propusieron una forma de clasificar la EC según el grado de severidad de la afección. En orden decreciente de severidad: ectasia difusa de dos o tres vasos (tipo I), enfermedad difusa en un vaso y enfermedad localizada en otro vaso (tipo II), ectasia difusa en un vaso (tipo III) y ectasia segmentaria localizada (tipo IV) ${ }^{4}$. En cuanto a la presentación clínica de estos pacientes, la angina crónica estable tiende a ser la más común 7 . Los síndromes coronarios agudos, tanto con elevación como sin elevación del segmento ST, pueden ocurrir por la alteración del flujo sanguíneo secundario a la embolización distal o la oclusión trombótica de un segmento ectásico ${ }^{8,9}$.

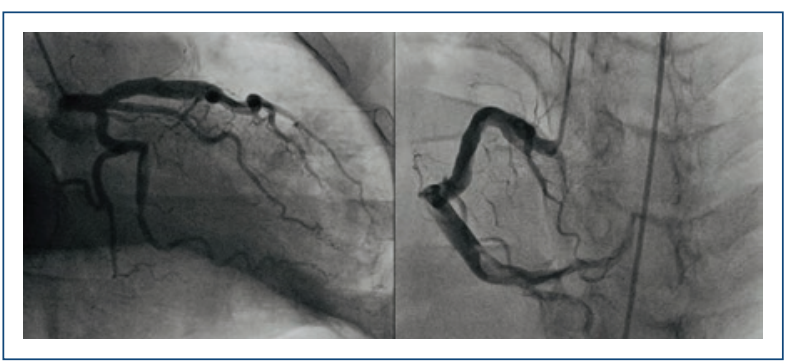

Figura 2. Coronariografía con presencia de arterias ectásicas.

En esta ocasión al presentarse como una taquicardia con complejo ancho, el correcto diagnóstico del origen de la taquicardia se vuelve esencial para la toma de decisiones, existiendo algoritmos planteados para lograr hacer una diferenciación entre taquicardia supraventricular y TV, empleando los criterios diagnósticos tanto de Brugada como de Verckei ${ }^{10,11}$. El electrocardiograma del paciente muestra una onda $R$ inicial en la derivación aVR, así como un ancho de esta mayor a $40 \mathrm{~ms}$, cumpliendo dos criterios de Verckei para ser catalogada como TV. Por otro lado, si bien de acuerdo con los criterios de Brugada no hay ausencia de RS en todas las precordiales, el tiempo $\mathrm{R}$ a $\mathrm{S}$ es menor de $100 \mathrm{~ms}$, no hay disociación V-A, cumple con otros criterios morfológicos en V1 y V6 de TV en presencia de bloqueo de rama derecha. 
El término «tormenta eléctrica» describe un estado de inestabilidad eléctrica del corazón caracterizado por la agrupación de episodios recurrentes de TV o fibrilación ventricular (FV) en un corto periodo de tiempo. En los últimos años, el implante de un desfibrilador automático implantable (DAI) ha mejorado significativamente la supervivencia de los pacientes con TV/FV. Sin embargo, las tormentas eléctricas se asocian a una elevada mortalidad y morbilidad, y tienen un impacto negativo en los desenlaces clínicos a largo plazo ${ }^{15}$. Aunque no existe un consenso sobre la definición de una tormenta eléctrica, generalmente se acepta que la aparición de $>2$ episodios de TV separada/o $>2$ episodios de FV $0>3$ terapias apropiadas del DAI para TV/FV en un periodo de $24 \mathrm{~h}$ comprenden un episodio de tormenta eléctrica.

La EC se ha asociado a un fenómeno de flujo coronario lento secundario, caracterizado por el retraso distal en la opacificación del vaso coronario en ausencia de estenosis epicárdica coronaria significativa ${ }^{6}$. El mecanismo patogénico no se conoce completamente. Clínicamente, este fenómeno ocurre en hombres jóvenes y fumadores en pacientes admitidos como síndrome coronario agudo $^{7}$ y se ha asociado a arritmias malignas y muerte cardiaca súbita ${ }^{12-14}$.

Consideramos que el fenómeno de flujo coronario lento cobró un papel pivote en la fisiopatología de la tormenta eléctrica en el caso expuesto. Mucho queda por elucidar sobre la interrelación que existe entre la $E C$, las dinámicas de flujo coronario y la carga arrítmica en el contexto del síndrome coronario isquémico. Hasta nuestro mejor entendimiento, este es el primer caso que reporta la asociación directa de EC y tormenta eléctrica.

\section{Financiación}

Ninguna.

\section{Conflicto de intereses}

Ninguno.

\section{Responsabilidades éticas}

Protección de personas y animales. Los autores declaran que para esta investigación no se han realizado experimentos en seres humanos ni en animales.

Confidencialidad de los datos. Los autores declaran que han seguido los protocolos de su centro de trabajo sobre la publicación de datos de pacientes.

Derecho a la privacidad y consentimiento informado. Los autores han obtenido el consentimiento informado de los pacientes y/o sujetos referidos en el artículo. Este documento obra en poder del autor de correspondencia.

\section{Bibliografía}

1. Hartnell GG, Parnell BM, Pridie RB. Coronary artery ectasia. Its exercise induced myocardial ischemia in isolated coronary artery prevalence and clinical significance in 4,993 patients. Br Heart J. 1985;54:392-95.

2. Mangina A, Cokkinos D. Coronary artery ectasia: imaging, functional assessment and clinical implications. Eu Heart J. 2006;27:1026-31.

3. Morgagni JB. De sedlbus, et causis morborum per anatomen indagatis. 1761. Tomus primus, Liber II, Epist 27, Article 28, Venetiis.

4. Markis JE, Joffe CD, Cohn PF, Feen DJ, Herman MV, Gorlin R. Clinical significance of coronary arterial ectasia. Am J Cardiol. 1976;37:217-22.

5. Pinar-Bermúdez E, López-Palop R, Martínez-Luengas IL, Cortés-Sánchez R, Carrillo-Sáez P, Rodríguez-Carreras R, et al. Ectasia coronaria: prevalencia, características clínicas y angiográficas. Rev Esp Cardiol. 2003:56:473-9.

6. Beltrame JF, Limaye SB, Horowitz JD. The coronary slow flow phenomenon-a new coronary microvascular disorder. Cardiology. 2002; 97:197-202.

7. Aboeata AS, Sontineni SP, Alla VM, Esterbrooks DJ. Coronary artery ectasia: current concepts and interventions. Front Biosci Elite Ed. 2012;4:300-10.

8. Rath S, Har-Zahav Y, Battler A, Agranat O, Rotstein Z, Rabinowitz B, et al. Fate of nonobstructive aneurysmatic coronary artery disease: angiographic and clinical followup report. Am Heart J. 1985;109(4):785-91.

9. Akyürek O, Berkalp B, Sayin T, Kumbasar D, Kervancioğlu C, Oral D. Altered coronary flow properties in diffuse coronary artery ectasia. Am Heart J. 2003;145(1):66-72.

10. Vereckei A, Duray G, Szénási G, Altemose GT, Miller JM. New algorithm using only lead aVR for differential diagnosis of wide QRS complex tachycardia. Heart Rhythm. 2008;5(1):89-98

11. Brugada P, Brugada J, Mont L, Smeets J, Andries EW. A new approach to the differential diagnosis of a regular tachycardia with a wide QRS complex. Circulation. 1991;83(5):1649-59.

12. Wozakowska-Kapłon B, Niedziela J, Krzyzak P, Stec S. Clinical manifestations of slow coronary flow from acute coronary syndrome to serious arrhythmias. Cardiol J. 2009;16:462-8.

13. Saya S, Hennebry TA, Lozano P, Lazzara R, Schechter E. Coronary slow flow phenomenon and risk for sudden cardiac death due to ventricular arrhythmias: a case report and review of literature. Clin Cardiol. 2008;31:352-5.

14. Maruyama M, Yamamoto T. Electrical storms: Recent advances. En: Kibos AS, Knight BP, editores. Cardiac arrhythmias: From basic mechanism to state-ofthe-art management. Londres: Springer-Verlag; 2014. pp. 285-92.

15. Gao D, Sapp JL. Electrical storm: definitions, clinical importance, and treatment. Curr Opin Cardiol. 2013;28:72-9. 